Original article

\title{
Exploring the association between melanoma and glioma risks
}

\author{
Peter M. Scarbrough $\mathrm{PhD}^{\mathrm{a}}$, Igor Akushevich $\mathrm{PhD}^{\mathrm{a}}$, Margaret Wrensch $\mathrm{PhD}^{\mathrm{b}}$, Dora Il'yasova $\mathrm{PhD}^{\mathrm{a}, \mathrm{c}, *}$ \\ ${ }^{a}$ Duke Cancer Institute, Duke University Medical Center, Durham, NC \\ ${ }^{\mathrm{b}}$ Department of Neurological Surgery, University of California, San Francisco, San Francisco \\ ${ }^{\mathrm{c}}$ Division of Epidemiology and Biostatistics, School of Public Health, Georgia State University, Atlanta
}

\section{A R T I C L E I N F O}

\section{Article history:}

Received 16 August 2013

Accepted 12 February 2014

Available online 4 March 2014

\section{Keywords:}

SEER

Epidemiology

Melanoma

Glioma

Glioblastoma

\begin{abstract}
A B S T R A C T
Purpose: Gliomas are one of the most fatal malignancies, with largely unknown etiology. This study examines a possible connection between glioma and melanoma, which might provide insight into gliomas' etiology.

Methods: Using data provided by the Surveillance, Epidemiology, and End Results program from 1992 to 2009, a cohort was constructed to determine the incidence rates of glioma among those who had a prior diagnosis of invasive melanoma. Glioma rates in those with prior melanoma were compared with those in the general population.

Results: The incidence rate of all gliomas was greater among melanoma cases than in the general population: 10.46 versus 6.13 cases per 100,000 person-years, standardized incidence ratios $=1.42(1.22-1.62)$. The female excess rate was slightly greater (42\%) than that among males (29\%). Sensitivity analyses did not reveal evidence that radiation treatment of melanoma is responsible for the detected gap in the rates of gliomas. Conclusions: Our analysis documented increased risk of glioma among melanoma patients. Because no common environmental risk factors are identified for glioma and melanoma, it is hypothesized that a common genetic predisposition may be responsible for the detected association.
\end{abstract}

(C) 2014 Elsevier Inc. All rights reserved.

\section{Introduction}

Gliomas are tumors of neuroepithelial tissue, arising from glial cell lineage, and make up the largest percentage of all malignant central nervous system (CNS) tumors (77\%) [1-3]. These cancers are rare, having a population-based incidence rate in the United States of approximately six cases per 100,000 person-years for adult glioma [1-6]. However, although these tumors are rare, they present one of the most fatal malignancies. Most subtypes of glioma tend to be aggressive and difficult to treat, with median survival below 2 years, especially in those aged 60 years and older [1]. Understanding glioma, etiology would provide a key to prevention and advancement in treatment [7]. However, despite several decades of etiologic research, the epidemiology of glioma has provided very few clues. Besides demographic factors such as gender (male), race (Caucasian), and age, the only established nongenetic risk factor for glioma is high-dose radiation [1,4,8,9]. An emerging line of etiologic glioma research studies connections between glioma and other disorders. For example, there is now a reported inverse association between the risk of glioma and a history of atopic conditions, such

\footnotetext{
* Corresponding author. School of Public Health, GSU, PO Box 3995, Atlanta, GA 30302. Tel/fax: 404-413-1135.

E-mail address: dilyasova@gsu.edu (D. Il'yasova).
}

as allergies, asthma, and eczema [10]. Such connections between different conditions may be helpful in elucidating common etiologic components. In this regard, a suspected association between melanoma and glioma represents a possibility to discover a common etiologic link between the two disorders and thereby to provide insight into glioma etiology.

An association between melanoma and glioma was first suggested by the observation of a familial association between melanoma and gliomas [11-13], which was later confirmed by the description of the melanoma-astrocytoma syndrome, involving deletions at genetic loci coding for a number of important cell cycle control proteins [14]. There were also two later analyses, further supporting the connection between glioma and melanoma: (i) melanoma occurred more frequently than expected among firstdegree relatives of glioma patients [15] and (ii) the families with glioma had a significant excess of melanoma cases [16]. We also found an association between self-reported history of melanoma and the high-grade glioma in the analysis of a case-control study; this was an unpublished incidental finding, as the main analysis explored gene-environment interactions [17]. Because we could not validate the self-reported history of melanoma in this previous study, we turned to the National Cancer Institute Surveillance Epidemiology and End Results (SEER) database, to test the hypothesis that glioma rates are greater among melanoma cases compared with those in the general population. 


\section{Materials and methods}

\section{Data source}

For this study, population-based data from the National Cancer Institute's SEER program were used, which cover approximately $26 \%$ of the United States' population [18]. Seventeen SEER registry databases were used, including data from 1992 to 2009, released on April 2010, but excluding cases impacted by Hurricane Katrina in Louisiana [19]. This study was limited to adult glioma (age $>20$ years).

Definition of glioma, glioblastoma, and melanoma cases and selection criteria

Glioma cases were defined using information provided by the Central Brain Tumor Registry of the United States [20]. Data for glioma cases were collected over the period of 1992-2009, using the ICD-O-3 site codes (C70.0-C72.9) and histology codes 9380-9460 for all gliomas and only the histology codes of 9440 , 9441, and 9442 for glioblastoma (GB). Invasive melanoma cases were collected through the period of 1992-2009, using site codes (C44.0-C44.9) and ICD-O-3 histology codes 8720-8790. Only cases with confirmed histology of invasive melanoma and glioma were included in the analysis.

In analysis of other prior cancers, besides melanoma, ICD-O-3 site codes were used to define the other prior cancer sites in the database, for cohort selection. The International Classification of Diseases (ICD)-O-3 site codes used for breast cancer were C50.0-C50.9, for colon cancer the codes used were (C18.0, C18.2-C18.9, and C19.9), and the site code C61.9 was used for prostate cancer.

Starting in 1992, any individual with an incidence of invasive melanoma was entered into a cohort and followed until an incidence of glioma (in which event they were labeled as a case of "glioma with prior melanoma"), or until censoring (death or reaching the end of the study follow-up period, 2009). After an individual's entry into the study cohort, if multiple primaries occurred during follow-up, besides glioma, the individual was still counted as a case as long as they also had an incidence of glioma.

The time period for this analysis starting at 1992 was selected to avoid an increase in glioma detection because of the introduction of magnetic resonance imaging technology into the clinic [6]; previously published analysis by Dubrow and Darefsky demonstrated a lack of increase in observed glioma incidence since 1992 [21].

\section{Study variables}

Race was recoded as a three-leveled variable: white, black, and other. Age-specific incidence rates were calculated for those with ages between 20 and 84 years. The upper limit for age inclusion was 84 years, because SEER population data do not specify age beyond 85 years of age. Therefore, the age-standardized rates could not be calculated for the unconditional incidence of glioma, melanoma, and GB, among those in ages above 84 .

\section{Model and statistical analysis}

All modeling and statistical analyses were performed in SAS, version 9.2 (SAS, Cary, NC). To calculate the rates of glioma, conditional on the incidence of a prior incidence of melanoma, invasive melanoma cases were included into a cohort and followed until the incidence of glioma or until censoring (reaching the end of the study period or death). Incidence of melanoma would qualify an individual for inclusion into the melanoma cohort, including melanoma diagnosis not being the first primary cancer. Any subsequent incidence of glioma was then counted as a case. The empirical agespecific rates $\left(\lambda_{a}\right)$ were calculated as a ratio of the numbers of cases $\left(n_{a}\right)$ to person-years $\left(P_{a}\right)$ at risk: $\lambda_{a}=n_{a} / P_{a}$. The standard error (SE) was calculated as $\sigma_{E}=\sqrt{\lambda_{a}\left(1-\lambda_{a}\right) / P_{a}}$. The age-adjusted rates (or directly standardized incidence rates) are calculated for age 20-84 years as $\lambda=\sum_{a=20}^{84} \lambda_{a} P_{a, \text { std }}\left(\sum_{a^{\prime}=20}^{84} P_{a^{\prime}, s t d}\right)^{-1}$, where $P_{a, \text { std }}$ are the age-specific counts of the standard population. The standard error for the age-adjusted rate is estimated assuming that the numbers of events observed in each age group are independent [22].

The population in the SEER areas was used for calculation of the unconditional rates, that is, glioma rates in the general population. Furthermore, we considered this population as standard and used for standardization of glioma rates in the melanoma cohort. It is important that we used the same population both for the denominator in calumniation of unconditional glioma rates and for age-standardization of glioma rates in the melanoma cohort, as this insures comparability of the rate in the general population and in the melanoma cohort. To estimate proportion of excess glioma cases in the melanoma cohort, standardized incidence ratios (SIRs) and their 95\% confidence intervals (CIs) were calculated as described previously [22]. SIRs were age-standardized and stratified by gender; race adjustment or stratification was not applicable as no glioma cases were identified among the melanoma cohort in non-white racial categories. To compare glioma rates in the melanoma cohort in the sensitivity analysis, we calculated $t$-statistics (a ratio of the rate difference and SE for this difference evaluated as the square root of the sum of their standard errors squared) and applied $t$ test. Because the estimates of rates were obtained based on the large number of cases, respective $t$ distributions with large degrees of freedom were well approximated by normal distributions.

\section{Results}

In this analysis, we identified 51,088 glioma cases and 242,471 invasive melanoma cases diagnosed between 1992 and 2009. Among the glioma cases, 29,702 (58.1\%) were GB. In the melanoma cohort, 192 incident glioma cases and 114 (59.4\%) incident GB cases were identified. The follow-up time ranged from 0 to 17.9 personyears, with a median follow-up time of 4.08 person-years.

Similar trends were observed in age and gender distributions for melanoma and glioma cases of all categories; specifically, the number of cases increased with age peaking at the seventh decade with slight decline at age 70 years or older (Table 1 ). The gender distribution of melanoma, unconditional glioma, and GB cases were similar (40\% female), with only a slightly lower observed proportion of females in the glioma with prior melanoma cohort (34\%). These results were expected, because both gliomas and melanomas are known to be less common in females than in males. White population accounted for most melanoma and all glioma and GB cases ( $\sim 90 \%)$ and all (100\%) of all glioma and GB cases with prior melanoma (Table 1 ).

As expected, the incidence rates (per 100,000 person-years) of melanoma, all gliomas, and GB increased with age (Table 2). Whereas the increase in melanoma rates was monotonic, the rates of all gliomas and of GB decreased after age 80. Also expected [21] were greater rates of glioma among men: specifically, men had approximately 1.4-fold greater incidence rate of all gliomas and of GB compared with women. Racial differences in melanoma and glioma were also in agreement with previous studies: rates of melanoma, all gliomas, and GB were greater among whites [21,23]. In summary, the results presented in Tables 1 and 2 display the expected findings, serving as an important quality control check for this analysis. 
Table 1

Gender, race and age distributions for glioma, melanoma, and glioma with prior melanoma cases. SEER cases were obtained from 1992 to 2009

\begin{tabular}{|c|c|c|c|c|}
\hline \multirow[t]{2}{*}{ Characteristic } & \multicolumn{4}{|c|}{$\%$ (number of cases) } \\
\hline & Melanoma & All gliomas & GB & $\begin{array}{l}\text { All gliomas } \\
\text { with prior } \\
\text { melanoma }\end{array}$ \\
\hline \multicolumn{5}{|c|}{ Age at diagnosis ${ }^{*}$} \\
\hline $20-29$ & $3.38(8202)$ & $5.90(3014)$ & $1.32(392)$ & $1.44(3)$ \\
\hline $30-39$ & $9.03(21,899)$ & $9.70(4955)$ & $3.22(955)$ & $1.92(4)$ \\
\hline $40-49$ & $15.2(36,895)$ & $14.4(7355)$ & $9.62(2857)$ & $8.17(17)$ \\
\hline $50-59$ & $18.5(44,892)$ & $19.8(10,130)$ & $20.8(6170)$ & $15.9(33)$ \\
\hline $60-69$ & $18.6(45,060)$ & $21.2(10,845)$ & $26.6(7902)$ & $20.2(42)$ \\
\hline $70-79$ & $18.3(44,420)$ & 19.4 (9927) & $25.9(7703)$ & $31.7(66)$ \\
\hline $80+$ & $17.0(41,103)$ & $9.52(4862)$ & $12.5(3723)$ & $20.7(43)$ \\
\hline \multicolumn{5}{|l|}{ Gender } \\
\hline Male & $58.5(141,880)$ & $57.25(29,249)$ & $58.2(17,277)$ & $65.9(137)$ \\
\hline Female & $41.5(100,591)$ & $42.75(21,839)$ & $41.8(12,425)$ & $34.1(71)$ \\
\hline \multicolumn{5}{|l|}{ Race } \\
\hline White & $92.5(224,237)$ & $89.4(45,674)$ & $90.6(26,919)$ & $100(208)$ \\
\hline Black & $2.08(5048)$ & $5.45(2785)$ & $5.20(1545)$ & $0(0)$ \\
\hline Other & $5.44(13,186)$ & 5.15 (2629) & $4.17(1238)$ & $0(0)$ \\
\hline
\end{tabular}

* For "prior melanoma," age category is determined by age at all glioma and GB diagnosis.

The unconditional (or general population) rates of glioma and GB incidence were compared with the incidence rates in those with a prior diagnosis of melanoma (Table 3, Fig. 1). For those with a prior diagnosis of melanoma, the unadjusted incidence rates for all gliomas were 1.7-fold greater than those of the general population: 10.46 versus 6.13 cases per 100,000 person-years, with $\sim 40 \%$ excess glioma cases in the melanoma cohort $(\mathrm{SIR}=1.42,95 \% \mathrm{CI}$, $1.22-1.62$ ). This gap was the largest for all gliomas at ages $20-29$ and 70 years or older (Fig. 1). Although the difference in the rates was more pronounced among men ( 16.0 vs. 7.51 cases per 100,000 person-years) as compared with women (6.38 vs. 5.31 cases per 100,000 person-years), the excess cases was greater among females ( $\sim 40 \%$ ) compared with males ( $30 \%$; Table 3 ). Overall, GB rates were approximately 1.3 -fold greater among melanoma cases compared with the general population (4.61 vs. 3.58 cases per 100,000 person-years), with $\sim 29 \%$ excess cases ( $\mathrm{SIR}=1.29: 95 \% \mathrm{CI}$, 1.18-1.40). Female GB cases accounted for most excess cases, although gender-specific SIRs were not statistically significant most likely because of decreased sample size.

Sensitivity analyses were performed to examine several assumptions. The main analysis included only invasive melanoma cases. We examined whether inclusion of all melanoma diagnosis would influence our results (Table 4). Our analysis indicates that the glioma rates did not change considerably when noninvasive melanoma cases were included in the analysis: 10.46 versus 9.88 cases per 100, 000 person-years $(P=.79)$. Furthermore, we examined the assumption that increased rates of glioma among melanoma cases may be due to radiation treatment of melanoma, as radiation is an established risk factor for glioma [8]. The rates among all the invasive cases of melanoma and for those who did not undergo radiation were virtually the same $(P=.98)$ (Table 4$)$. This suggests
Table 2

Incidence rates of invasive melanoma, all gliomas, and GB by demographic characteristics, SEER cases diagnosed in 1992-2009

\begin{tabular}{llll}
\hline Characteristic & \multicolumn{3}{l}{ Rate per 100,000 person-years (SE) } \\
\cline { 2 - 4 } & Melanoma & All gliomas & GB \\
\hline Age (y) & $5.15(0.06)$ & $1.90(0.03)$ & $0.25(0.01)$ \\
$20-29$ & $12.9(0.09)$ & $2.92(0.04)$ & $0.56(0.02)$ \\
$30-39$ & $21.6(0.11)$ & $4.31(0.05)$ & $1.68(0.03)$ \\
$40-49$ & $32.9(0.16)$ & $7.42(0.07)$ & $4.52(0.06)$ \\
$50-59$ & $51.1(0.24)$ & $12.3(0.12)$ & $8.97(0.10)$ \\
$60-69$ & $73.3(0.35)$ & $16.4(0.16)$ & $12.7(0.14)$ \\
$70-79$ & $93.0(0.46)$ & $11.0(0.16)$ & $8.43(0.14)$ \\
$80+$ & $27.4(0.06)$ & $6.13(0.03)$ & $3.52(0.02)$ \\
Overall: $20 \leq$ age $\leq 84$ & & & \\
Gender (age-adjusted) & $32.6(0.09)$ & $7.15(0.04)$ & $4.19(0.03)$ \\
Male & $22.4(0.07)$ & $5.13(0.04)$ & $2.88(0.03)$ \\
Female & & & \\
Race (age-adjusted) & $32.5(0.07)$ & $7.02(0.03)$ & $4.09(0.03)$ \\
White & $5.28(0.08)$ & $2.99(0.06)$ & $1.65(0.04)$ \\
$\quad$ Black &
\end{tabular}

* For "prior melanoma," age category is determined by age at all glioma and GB diagnosis.

that interventions on melanoma cases most likely did not influence the rate of glioma in the melanoma cohort.

Furthermore, we reasoned that the etiologic link between melanoma and glioma would be further supported if the reverse association was found to be true (i.e., if there was a positive between the incident rates of melanoma and a prior diagnosis for glioma). Therefore, we compared the incidence rate of melanoma in a cohort of those with a prior incidence of glioma to the general population and found elevated SIR of 3.01 (95\% CI, 2.28-3.74).

Additional sensitivity analysis was conducted to test the specificity of the association between glioma incidence and prior melanoma as opposed to other cancer sites. For this purpose, we tracked the incidence of glioma in cohorts in individuals with prior colon cancer in both sexes, prior breast cancer in women only, and prior prostate cancer in men only. The cohorts' effects were analyzed separately; the period of follow-up for each cohort and their censoring criteria were the same as in the melanoma cohort. None of the prior cancer sites investigated showed a positive association with incidence rate of glioma: SIRs were 0.89 (95\% CI, $0.77-1.01$ ) for colon, 0.88 for breast $(95 \% \mathrm{CI}, 0.78-0.98)$, and 1.01 (95\% CI, 0.93-1.09) for prostate cancers. Together, these findings demonstrate the specificity of the association between melanoma and glioma.

\section{Discussion}

The main finding of this analysis is increased incidence rate of gliomas among those with a prior incidence of melanoma compared with the general population (Table 3). Results from Tables 1 and 2 illustrate the consistency of this study with prior work $[7,21,24,25]$, supporting generalizability of our main finding.

Table 3

Incidence rates and standardized incidence ratios of all gliomas and GB among those with a prior diagnosis of invasive melanoma, SEER cases diagnosed in $1992-2009$

\begin{tabular}{|c|c|c|c|c|}
\hline \multirow[t]{2}{*}{ Characteristic } & \multicolumn{2}{|c|}{ Any glioma, given prior melanoma } & \multicolumn{2}{|c|}{ Only GB, given prior melanoma } \\
\hline & $\begin{array}{l}\text { Rate per } 100,000 \\
\text { person-years (SE) }[N]\end{array}$ & $\begin{array}{l}\text { SIR }(95 \% \mathrm{CI}) \\
\text { age-standardized }\end{array}$ & $\begin{array}{l}\text { Rate per } 100,000 \\
\text { person-years }(\mathrm{SE})[N]\end{array}$ & $\begin{array}{l}\text { SIR }(95 \% \mathrm{CI}) \\
\text { age-standardized }\end{array}$ \\
\hline Overall rates & $10.5(1.72)[192]$ & $1.42(1.22-1.62)$ & $4.61(0.84)[114]$ & $1.22(1.00-1.44)$ \\
\hline Male & $16.0(4.26)[161]$ & $1.29(1.07-1.51)$ & $6.02(2.02)[93]$ & $1.07(0.83-1.31)$ \\
\hline Female & $6.38(1.25)[31]$ & $1.42(1.07-1.77)$ & $3.46(1.08)[21]$ & $1.29(0.88-1.70)$ \\
\hline
\end{tabular}




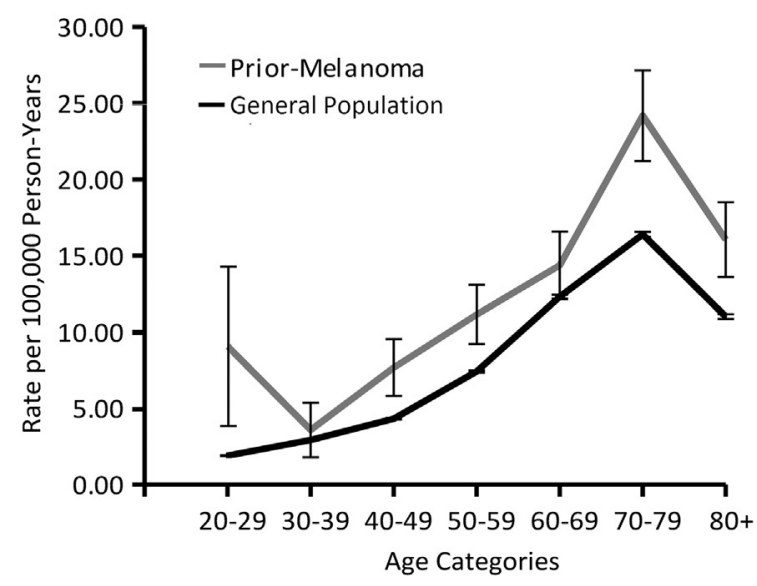

\begin{tabular}{|l|c|}
\hline Age & SIR $(95 \% \mathrm{CI})$ \\
\hline $20-29$ & $4.46(0.00-9.50)$ \\
\hline $30-39$ & $1.20(0.02-2.38)$ \\
\hline $40-49$ & $1.75(0.93-2.57)$ \\
\hline $50-59$ & $1.48(0.97-1.99)$ \\
\hline $60-69$ & $1.16(0.81-1.51)$ \\
\hline $70-79$ & $1.47(1.12-1.82)$ \\
\hline $80-84$ & $1.49(0.92-2.06)$ \\
\hline
\end{tabular}

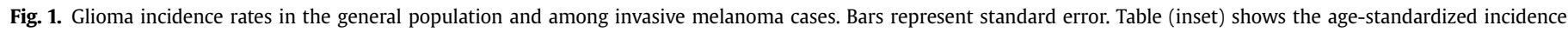

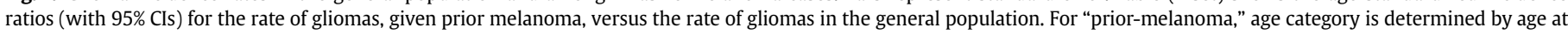
glioma diagnosis.

The detected association between melanoma and glioma appears to peak within two age groups, specifically at age $20-29$ and 70 years or older (Fig. 1). Four previous analyses of population-based data [26-29] examined the rates of second primary malignancies among patients with CNS tumors. We cannot compare our findings with the most recent SEER-based analysis, as it did not report the results on melanoma [26]. Of the three earlier studies, one SEER-based study did not find an increased risk for melanoma after a first primary cancer of the brain or CNS with SIR $=1.08,(95 \% \mathrm{CI}, 0.82-1.39)$ [27]. Two other studies found a positive association between melanoma and CNS tumors. One SEER-based study found an association between prior melanoma and subsequent CNS tumor, reporting an $\mathrm{SIR}=1.31$ (95\% CI, 1.13-1.51) [29] and another study used records of the Finnish Cancer Registry from 1953 to 1994 and found an association between prior CNS tumor and subsequent melanoma, reporting an SIR $=1.9$ (95\% CI, 1.0-3.1) [28]. Our results confirm these findings: SIR for the association of glioma with prior melanoma was 3.01 (95\% CI, 2.28-3.74). As for the fact that our results are inconsistent with one of the prior studies [27], we note that the results of this study could be influenced by small sample size (59 melanomas among patients with prior brain or CNS tumor) and by the vast heterogeneity of CNS tumors, both of which may mask an association between melanoma and subsequent glioma [27].

We also considered an assumption that greater glioma rates after melanoma diagnosis could be due to the increased surveillance among cancer patients. It is very unlikely that highgrade gliomas could be underdiagnosed in the general population, as these are rapidly growing tumors, often with severe symptoms. Yet, a potential detection and surveillance bias could exist for slowly growing tumors with wider variation of symptom severity. For example, consistent associations between slowly growing brain tumors and the indicators of higher socioeconomic status could signify a difference in the symptomatic threshold for diagnosis of these tumors and therefore, a possibility of a detection/surveillance bias. The analysis of such associations with different types of slowly growing brain tumors yielded mixed results [30]. Thus, we have not found strong arguments that the observed association between the two malignancies reflects a detection bias.

An association for two malignancies can be explained mainly by three nonmutually exclusive concepts: treatment-related increased risk of the second malignancy, common environmental factors, and common genetic predisposition. Our sensitivity analysis did not support the assumption that melanoma treatment is responsible for the detected increase in glioma rates (Table 4). Given what is known about the environmental risk factors for melanoma (the main risk factor is ultraviolet radiation) and glioma (the only established risk factor is ionizing radiation), it is unlikely that the environmental risk factors are shared between the two diseases. This is supported by the diverging time trends in melanoma (the

Table 4

Age-standardized incidence rates of all gliomas and GB in the general population and among different categories of melanoma cases

\begin{tabular}{|c|c|c|c|c|}
\hline \multirow[t]{2}{*}{ Characteristic } & \multicolumn{2}{|c|}{ Rates per 100,000 person-years (SE) $n=$ number of cases } & \multicolumn{2}{|l|}{$\operatorname{SIR}(95 \% \mathrm{CI})$} \\
\hline & All gliomas & GB & All gliomas & GB \\
\hline \multicolumn{5}{|c|}{ Invasive and noninvasive melanoma } \\
\hline Both genders (overall) & $9.88(1.38) n=324$ & $4.90(0.70) n=212$ & $1.40(1.24-1.56)$ & $1.28(1.10-1.46)$ \\
\hline Male only & $14.8(3.55) n=214$ & $6.29(1.71) n=144$ & $1.28(1.10-1.46)$ & $1.17(0.97-1.37)$ \\
\hline Female only & $6.42(1.01) n=110$ & $3.61(0.88) n=68$ & $1.40(1.13-1.67)$ & $1.26(0.93-1.59)$ \\
\hline \multicolumn{5}{|l|}{ Invasive melanoma } \\
\hline Both genders (overall) & $10.5(1.72) n=192$ & $4.61(0.84) n=114$ & $1.42(1.22-1.62)$ & $1.22(1.00-1.44)$ \\
\hline Male only & $16.0(4.26) n=161$ & $6.02(2.02) n=93$ & $1.29(1.07-1.51)$ & $1.07(0.83-1.31)$ \\
\hline Female only & $6.38(1.25) n=31$ & $3.46(1.08) n=21$ & $1.42(1.07-1.77)$ & $1.29(0.88-1.70)$ \\
\hline \multicolumn{5}{|c|}{ Invasive melanoma (only radiation tx cases) } \\
\hline Both genders (overall) & $7.54(25.5) n=9$ & $5.21(25.4) n=6$ & $1.49(0.45-2.53)$ & $1.57(0.32-2.82)$ \\
\hline Male only & $8.53(54.0) n=7$ & $5.27(53.9) n=4$ & $1.34(0.26-2.42)$ & $1.25(0.03-2.47)$ \\
\hline Female only & $4.71(51.2) n=2$ & $4.71(52.0) n=2$ & $1.40(0.00-3.34)$ & $1.98(0.00-4.72)$ \\
\hline \multicolumn{5}{|c|}{ Invasive melanoma (no radiation tx cases) } \\
\hline Both genders (overall) & $10.5(1.77) n=196$ & $4.55(0.87) n=119$ & $1.40(1.20-1.60)$ & $1.19(0.95-1.43)$ \\
\hline Male only & $16.3(4.41) n=127$ & $6.04(2.11) n=77$ & $1.27(1.03-1.51)$ & $1.04(0.80-1.28)$ \\
\hline Female only & $6.43(1.28) n=69$ & $3.42(1.10) n=42$ & $1.43(1.08-1.78)$ & $1.28(0.85-1.75)$ \\
\hline
\end{tabular}


rates of which had increased over the last several decades) and glioma (which have had no corresponding increase in rate) [21,31], suggesting different environmental determinants of these two malignancies. In this regard, an inverse association between atopic conditions including allergies with melanoma [10] might indicate that at least some exposures may influence the risk of both malignancies. However, allergies and atopic (or absence of those) conditions have a genetic component [32] and therefore, may also be indicative of a common genetic predisposition to certain malignancies.

Considering common genetic predisposition, the strongest evidence comes from other previously published studies, which identify common antigen expression and genetic alterations between melanoma and glioma [33-35]. The common chromosomal deletions found in melanoma-astrocytoma syndrome [14] also support a genetic link between melanoma and glioma. Another less evident connection between melanoma and glioma may involve ineffective DNA repair. Defective nucleotide excision repair (e.g., xeroderma pigmentosa) is a significant risk factor for melanoma, especially for melanomas, which occur early in life. As melanoma cases with defective DNA repair concentrate at younger ages, it is conceivable that defective DNA repair may underlie specifically the younger age peak of the association between melanoma and glioma. Although genetically predisposed cancer cases do tend to precipitate at a younger age, such cases do not exclusively become clinically apparent at a young age. If it is true that a common genetic predisposition involves alterations to one's DNA repair capacity, the increased rate of glioma in older ages with prior melanoma may reflect more subtle defects to DNA repair that require a much longer period of time to accumulate the genetic mutations necessary for a cell transformation.

We also considered a connection between melanoma and glioma via common molecular and cell signaling pathway mutations, such as p53 and epithelial growth factor receptor [36,37]. However, this seems to be a weak explanation, at least with respect to the known pathways and molecular signatures. For example, although some subtypes of glioma are known to be associated with p53 and epithelial growth factor receptor mutations, it is also known that most gliomas do not actually contain these mutations. Thus, it appears that if common predisposing genetic elements between the two diseases exist, they remain to be discovered.

Interestingly, in the melanoma cohort, the excess in GB cases seemed to be exclusively only female (Table 3 ). In part, this could be explained by low samples numbers (male: $n=93$ and female: $n=21$ ). However, males have historically higher rates of glioma and GB than women, and an emerging trend in the literature is that hormonal differences between genders may explain why. A recent meta-analysis of hormone replacement therapy and oral contraceptives found a protective effect in women for glioma incidence (hormone replacement therapy: relative risk $=0.68,95 \% \mathrm{CI}$, $0.58-0.81$; oral contraceptives: relative risk $=0.707,95 \% \mathrm{CI}$, 0.604-0.828) [38]. Although the constituents of these therapies are primarily estrogen and progesterone, this suggests that the hormones may have an endogenous protective effect in women, potentially explaining the historically elevated glioma rates in men, also observed in this study. Thus, we interpret the excess GB cases in women in the melanoma cohort to be especially intriguing. If replicated in another study, they would suggest that the genetic predisposing factors to glioma may obviate the protective effects of hormones or some other unknown gender-based difference.

Overall, the data in the study show that prior diagnosis of melanoma is positively associated with the incidence of glioma. Taking into account the familial connection between the two malignancies and lack of common environmental risk factors, it is most likely that the detected association reflects some common genetic predisposition, which could have age-specific effects. As the main direction of current glioma research lies within the exploration of genetic predisposition, our results provide direction for a search of possible targets that can be pursued by future studies in glioma.

\section{Acknowledgments}

This work was supported by National Institutes of Health R25CA12693804 and R01CA139020.

\section{References}

[1] Central Brain Tumor Registry of the United States. Central Brain Tumor Registry of The United States. Available at: http://www.CBTRUS.org; 2013 [Accessed May 28, 2013].

[2] Kleihues P, Cavenee WK. Tumors of the central nervous system: pathology and genetics. Lyon, France: International Agency for Research on Cancer; 1995.

[3] Schwartzbaum JA, Fisher JL, Aldape KD, Wrensch M. Epidemiology and molecular pathology of glioma. Nat Clin Pract Neurol 2006;2:494-503.

[4] Ries LAG, Eisner MP, Kosary CL, Hankey BF, Miller BA, Clegg L, et al. SEER cancer statistics review, 1975-2002. Bethesda, Md: National Cancer Institute 2005.

[5] Surawicz TS, McCarthy BJ, Kupelian V, Jukich PJ, Bruner JM, Davis FG. Descriptive epidemiology of primary brain and CNS tumors: results from the Central Brain Tumor Registry of the United States, 1990-1994. Neuro Oncol 1999:1:14-25.

[6] Deorah S, Lynch CF, Sibenaller ZA, Ryken TC. Trends in brain cancer incidence and survival in the United States: Surveillance, Epidemiology, and End Results Program, 1973 to 2001. Neurosurg Focus 2006;20:E1.

[7] Bondy ML, Scheurer ME, Malmer B, Barnholtz-Sloan JS, Davis FG, Il'yasova D, et al. Brain Tumor Epidemiology Consortium. Brain tumor epidemiology: consensus from the Brain Tumor Epidemiology Consortium. Cancer 2008:113:1953-68.

[8] Ron E. Cancer risks from medical radiation. Health Phys 2003;85:47-59.

[9] Thompson DE, Mabuchi K, Ron E, Soda M, Tokunaga M, Ochikubo S, et al. Cancer incidence in atomic bomb survivors. Part II: solid tumors, 1958-1987. Radiat Res 1994;137:S17-67.

[10] Linos E, Raine T, Alonso A, Michaud D. Atopy and risk of brain tumors: a metaanalysis. J Natl Cancer Inst 2007:99:1544-50.

[11] Kaufman DK, Kimmel DW, Parisi JE, Michels VV. A familial syndrome with cutaneous malignant melanoma and cerebral astrocytoma. Neurology 1993:43:1728-31

[12] Azizi E, Friedman J, Pavlotsky F, Iscovich J, Bornstein A, Shafir R, et al. Familial cutaneous malignant melanoma and tumors of the nervous system. A hereditary cancer syndrome. Cancer 1995;76:1571-8.

[13] Paunu N, Pukkala E, Laippala P, Sankila R, Isola J, Miettinen H, et al. Cancer incidence in families with multiple glioma patients. Int $\mathrm{J}$ Cancer 2002;97:819-22.

[14] Bahuau M, Vidaud D, Jenkins RB, Bièche I, Kimmel DW, Assouline B, et al Germ-line deletion involving the INK4 locus in familial proneness to melanoma and nervous system tumors. Cancer Res 1998;58:2298-303.

[15] Scheurer ME, Etzel CJ, Liu M, El-Zein R, Airewele GE, Malmer B, et al. Aggregation of cancer in first-degree relatives of patients with glioma. Cancer Epidemiol Biomarkers Prev 2007;16:2491-5.

[16] Scheurer ME, Etzel CJ, Liu M, Barnholtz-Sloan J, Wiklund F, Tavelin B, et al GLIOGENE Consortium. Familial aggregation of glioma: a pooled analysis. Am J Epidemiol 2010;172:1099-107.

[17] McCarthy BJ, Rankin K, Il'yasova D, Erdal S, Vick N, Ali-Osman F, et al. Assessment of type of allergy and antihistamine use in the development of glioma. Cancer Epidemiol Biomarkers Prev 2011;20:370-8.

[18] Hankey BF, Ries LA, Edwards BK. The surveillance, epidemiology, and end results program: a national resource. Cancer Epidemiol Biomarkers Prev 1999;8:1117-21.

[19] Surveillance, Epidemiology, and End Results (SEER) Program. SEER*Stat database: SEER 17 registries research data and Hurricane Katrina impacted Louisiana cases. Available at: http://www.seer.cancer.gov; 2010 [Accessed May 28, 2013].

[20] Central Brain Tumor Registry of the United States. Supplement report: primary brain tumors in the United States, 2004. Hinsdale, IL: Published by the Central Brain Tumor Registry of the United States; 2008.

[21] Dubrow R, Darefsky AS. Demographic variation in incidence of adult glioma by subtype, United States, 2002-2007. BMC Cancer 2011;11:325.

[22] Breslow NE, Day NE. Statistical methods in cancer research. Volume II-The design and analysis of cohort studies. IARC Sci Publ; 1987:1-406.

[23] Watson M, Johnson CJ, Chen VW, Thomas CC, Weir HK, Sherman R, et al. Melanoma surveillance in the United States: overview of methods. J Am Acad Dermatol 2011:65:S6-16.

[24] Akushevich I, Kravchenko J, Ukraintseva S, Arbeev K, Yashin AI. Time trends of incidence of age-associated diseases in the US elderly population: Medicarebased analysis. Age Ageing 2013:42:494-500 
[25] Purdue MP, Freeman LE, Anderson WF, Tucker MA. Recent trends in incidence of cutaneous melanoma among US Caucasian young adults. J Invest Dermatol 2008;128:2905-8.

[26] Strodtbeck K, Sloan A, Rogers L, Fisher PG, Stearns D, Campbell L, et al. Risk of subsequent cancer following a primary CNS tumor. J Neurooncol 2013;112:285-95.

[27] Inskip PD. Multiple primary tumors involving cancer of the brain and central nervous system as the first or subsequent cancer. Cancer 2003;98:562-70.

[28] Spanogle JP, Clarke CA, Aroner S, Swetter SM. Risk of second primary malignancies following cutaneous melanoma diagnosis: a population-based study. J Am Acad Dermatol 2010;62:757-67.

[29] Salminen E, Pukkala E, Teppo L. Second cancers in patients with brain tumours-impact of treatment. Eur J Cancer 1999;35:102-5.

[30] Inskip PD, Tarone RE, Hatch EE, Wilcosky TC, Fine HA, Black PM, et al. Sociodemographic indicators and risk of brain tumours. Int $\mathrm{J}$ Epidemiol 2003;32:225-33.

[31] McCarthy BJ, Propp JM, Davis FG, Burger PC. Time trends in oligodendroglial and astrocytic tumor incidence. Neuroepidemiology 2008;30:34-44.

[32] Kayserova J, Sismova K, Zentsova-Jaresova I, Katina S, Vernerova E, Polouckova A, et al. A prospective study in children with a severe form of atopic dermatitis: clinical outcome in relation to cytokine gene polymorphisms. J Investig Allergol Clin Immunol 2012;22:92-101.

[33] Solomon DA, Kim JS, Cronin JC, Sibenaller Z, Ryken T, Rosenberg SA, et al. Mutational inactivation of PTPRD in glioblastoma multiforme and malignant melanoma. Cancer Res 2008;68:10300-6.

[34] Kuan CT, Wakiya K, Keir ST, Li J, Herndon 2nd JE, Pastan I, et al. Affinitymatured anti-glycoprotein NMB recombinant immunotoxins targeting malignant gliomas and melanomas. Int J Cancer 2011;129:111-21.

[35] Killela PJ, Reitman ZJ, Jiao Y, Bettegowda C, Agrawal N, Diaz Jr LA, et al. TERT promoter mutations occur frequently in gliomas and a subset of tumors derived from cells with low rates of self-renewal. Proc Natl Acad Sci U S A 2013;110:6021-6.

[36] Scharti M, Wilde B, Laisney JA, Taniguchi Y, Takeda S, Meierjohann S, A mutated EGFR is sufficient to induce malignant melanoma with genetic background-dependent histopathologies. J Invest Dermatol 2010;130:249-58.

[37] Rhim KJ, Hong SI, Hong WS, Lee SY, Lee DS, Jang JJ. Aberrant expression of p53 gene product in malignant melanoma. J Korean Med Sci 1994:9:376-81.

[38] Qi ZY, Shao C, Zhang X, Hui GZ, Wang Z. Exogenous and endogenous hormones in relation to glioma in women: a meta-analysis of 11 case-control studies. PLoS One 2013;16:e68695. 